OPEN ACCESS

Edited by:

Giovanni Raffa

University of Messina, Italy

Reviewed by:

Cesare Zoia,

Fondazione IRCCS Policlinico San

Matteo, Italy

Philipp Hendrix

Geisinger Health System

United States

*Correspondence:

Sally Rosario Hazem

sally.hazem@nhs.net

Mariam Awan

m.awan1@nhs.net

tThese authors have contributed equally to this work

Specialty section:

This article was submitted to Neuro-Oncology and Neurosurgical

Oncology,

a section of the journal

Frontiers in Neurology

Received: 24 December 2020

Accepted: 29 January 2021

Published: 10 March 2021

Citation:

Hazem SR, Awan M, Lavrador JP

Patel S, Wren HM, Lucena O,

Semedo C, Irzan H, Melbourne A

Ourselin S, Shapey J,

Kailaya-Vasan A, Gullan R, Ashkan K,

Bhangoo $R$ and Vergani $F$ (2021)

Middle Frontal Gyrus and Area 55b:

Perioperative Mapping and Language Outcomes. Front. Neurol. 12:646075.

doi: 10.3389/fneur.2021.646075

\section{Middle Frontal Gyrus and Area 55b: Perioperative Mapping and Language Outcomes}

\author{
Sally Rosario Hazem ${ }^{1,2 * t}$, Mariam Awan ${ }^{1,2 * t}$, Jose Pedro Lavrador ${ }^{1,2 \dagger}$, Sabina Patel ${ }^{1,2}$, \\ Hilary Margaret Wren ${ }^{1}$, Oeslle Lucena ${ }^{3}$, Carla Semedo ${ }^{3,4}$, Hassna Irzan ${ }^{3,4}$, \\ Andrew Melbourne ${ }^{3,4}$, Sebastien Ourselin ${ }^{3}$, Jonathan Shapey ${ }^{1,2,3}$, Ahilan Kailaya-Vasan ${ }^{1,2}$, \\ Richard Gullan ${ }^{1}$, Keyoumars Ashkan ${ }^{1,2}$, Ranjeev Bhangoo ${ }^{1,2}$ and Francesco Vergani ${ }^{1,2}$ \\ 1 Department of Neurosurgery, King's College Hospital National Health Service Foundation Trust, London, United Kingdom, \\ ${ }^{2}$ King's Neuro Lab, Department of Neurosurgery, King's College Hospital National Health Service Foundation Trust, London, \\ United Kingdom, ${ }^{3}$ School of Biomedical Engineering and Imaging Sciences, King's College London, London, \\ United Kingdom, ${ }^{4}$ Department of Medical Physics and Biomedical Engineering, University College London, London, \\ United Kingdom
}

Background: The simplistic approaches to language circuits are continuously challenged by new findings in brain structure and connectivity. The posterior middle frontal gyrus and area 55b (pFMG/area55b), in particular, has gained a renewed interest in the overall language network.

Methods: This is a retrospective single-center cohort study of patients who have undergone awake craniotomy for tumor resection. Navigated transcranial magnetic simulation (nTMS), tractography, and intraoperative findings were correlated with language outcomes.

Results: Sixty-five awake craniotomies were performed between 2012 and 2020, and 24 patients were included. nTMS elicited 42 positive responses, $76.2 \%$ in the inferior frontal gyrus (IFG), and hesitation was the most common error (71.4\%). In the pMFG/area55b, there were seven positive errors (five hesitations and two phonemic errors). This area had the highest positive predictive value (43.0\%), negative predictive value (98.3\%), sensitivity (50.0\%), and specificity (99.0\%) among all the frontal gyri. Intraoperatively, there were 33 cortical positive responses-two $(6.0 \%)$ in the superior frontal gyrus (SFG), 15 (45.5\%) in the MFG, and 16 (48.5\%) in the IFG. A total of 29 subcortical positive responses were elicited-21 in the deep IFG-MFG gyri and eight in the deep SFG-MFG gyri. The most common errors identified were speech arrest at the cortical level (20 responses-13 in the IFG and seven in the MFG) and anomia at the subcortical level (nine patients - eight in the deep IFG-MFG and one in the deep MFG-SFG). Moreover, 83.3\% of patients had a transitory deterioration of language after surgery, mainly in the expressive component $(p=0.03)$. An increased number of gyri with intraoperative positive responses were related with better preoperative $(p=0.037)$ and worse postoperative $(p=0.029)$ outcomes. The involvement of the SFG-MFG subcortical area was related with worse language outcomes 
$(p=0.037)$. Positive nTMS mapping in the IFG was associated with a better preoperative language outcome $(p=0.017)$, relating to a better performance in the expressive component, while positive mapping in the MFG was related to a worse preoperative receptive component of language $(p=0.031)$.

Conclusion: This case series suggests that the posterior middle frontal gyrus, including area 55b, is an important integration cortical hub for both dorsal and ventral streams of language.

Keywords: area 55b, language mapping, speech arrest, perioperative mapping, DTI, TMS, language network, nTMS

\section{INTRODUCTION}

Previous models of parcellation of the cerebral cortex have been proposed based on cytoarchitectonic $(1,2)$, myeloarchitectonic $(3,4)$, or functional characteristics of the different cerebral cortical areas $(5,6)$.

More recently, a new mapping of the human cortex has been described, using a multi-modal gradient-based parcellation approach (7). One of the novelties of this approach has been the identification of new cortical areas with a distinctive myelo/cytoarchitectonic and functional profile. A particularly interesting region is the frontal area 55b. Initially noted by Hopf in 1956 (4), this area is located at the posterior aspect of the middle frontal gyrus (MFG) and is delimited by the frontal eye field (FEF) superiorly, the premotor eye field (PEF) inferiorly, the primary motor cortex and the ventral motor cortex posteriorly, and by the prefrontal areas anteriorly $(7,8)$. Area 55b appears to be lightly myelinated and lies between moderately myelinated areas (i.e., FEF above and PEF below) and anteriorly to heavily myelinated areas (i.e., primary motor cortex). It has been described to be involved in various language production tasks and fluency of speech $(7,9,10)$. These findings are responsible for the renewed interest in the contribution of the posterior MFG to the overall language network.

Techniques of brain mapping that have evolved to increase the extent and safety of tumor resection in eloquent areas of the brain (11) have the unique advantage of testing different functions of specific cortical areas and networks at the individual level (12). Direct electrical stimulation (DES) at the cortical and subcortical levels is the gold standard for intraoperative mapping, defining the functional borders of resection in glioma surgery (13-15). In addition, navigated transcranial magnetic stimulation (nTMS) has emerged over the past decade as a useful adjunct for the preoperative mapping of motor (16-21) and language (22-27) areas of the brain.

In the present paper, we reviewed the results obtained by combining DES and nTMS in the functional assessment of the middle frontal gyrus and area $55 \mathrm{~b}$ in a series of patients undergoing awake surgery for brain tumors. In addition, we evaluated the potential relationship between preoperative and intraoperative language mapping and between the assessment of language performed prior to and following surgery, with a view to assess the relative contribution of the MFG on the language outcome. The preoperative and intraoperative findings are reviewed, and the potential role of these areas as part of the language network is discussed.

\section{MATERIALS AND METHODS}

This is a retrospective single-center cohort study of nonconsecutive patients admitted with language eloquent tumors for surgical treatment from January 2012 to January 2020. The inclusion criteria for the current study were age above 18 years old, awake craniotomy with DES for language mapping, and a tumor located in the dominant frontal lobe. Hemispheric dominance was assessed with the Edinburgh Handedness Inventory scale. The exclusion criteria included failed awake craniotomy and awake craniotomy for non-language mapping purposes.

\section{Language Assessment}

The preoperative and postoperative assessments were performed using the Sheffield Aphasia Screening Test for Acquired Language Disorder (SST) (28). This test was applied by the same speech and language therapist responsible for the intraoperative language testing. The patients were interviewed pre- and postoperatively to assess their communication abilities in conversational speech. Subtle subjective changes pertaining to comprehension, speech, reading, or writing abilities affecting daily living were evaluated. Where relevant, additional subtests were administered from the Mount Wilga Higher Level Language Test (29). The language errors were divided into speech arrest, hesitation, fluency disturbance, repetition disturbance, semantic paraphasia, and anomia.

\section{Intraoperative Mapping}

An asleep-awake-asleep craniotomy was performed in all the included patients. Low-frequency intraoperative stimulation according to the Penfield technique (30) was performed. Then, $50-\mathrm{Hz}$ biphasic square wave pulses of $1-\mathrm{ms}$ duration were applied using a constant current stimulator (ISIS Neurostimulator; Inomed Medizintechnik $\mathrm{GmbH}$ ). The current threshold used for brain mapping was the minimal current responsible for speech arrest during the counting task (two out of three attempts) or the highest current non-responsible for after-discharges. The exposed cortical area was mapped with one stimulation area every $2-3 \mathrm{~cm}$ at least three times per language task. The selection of the intraoperative tasks were performed according to the 

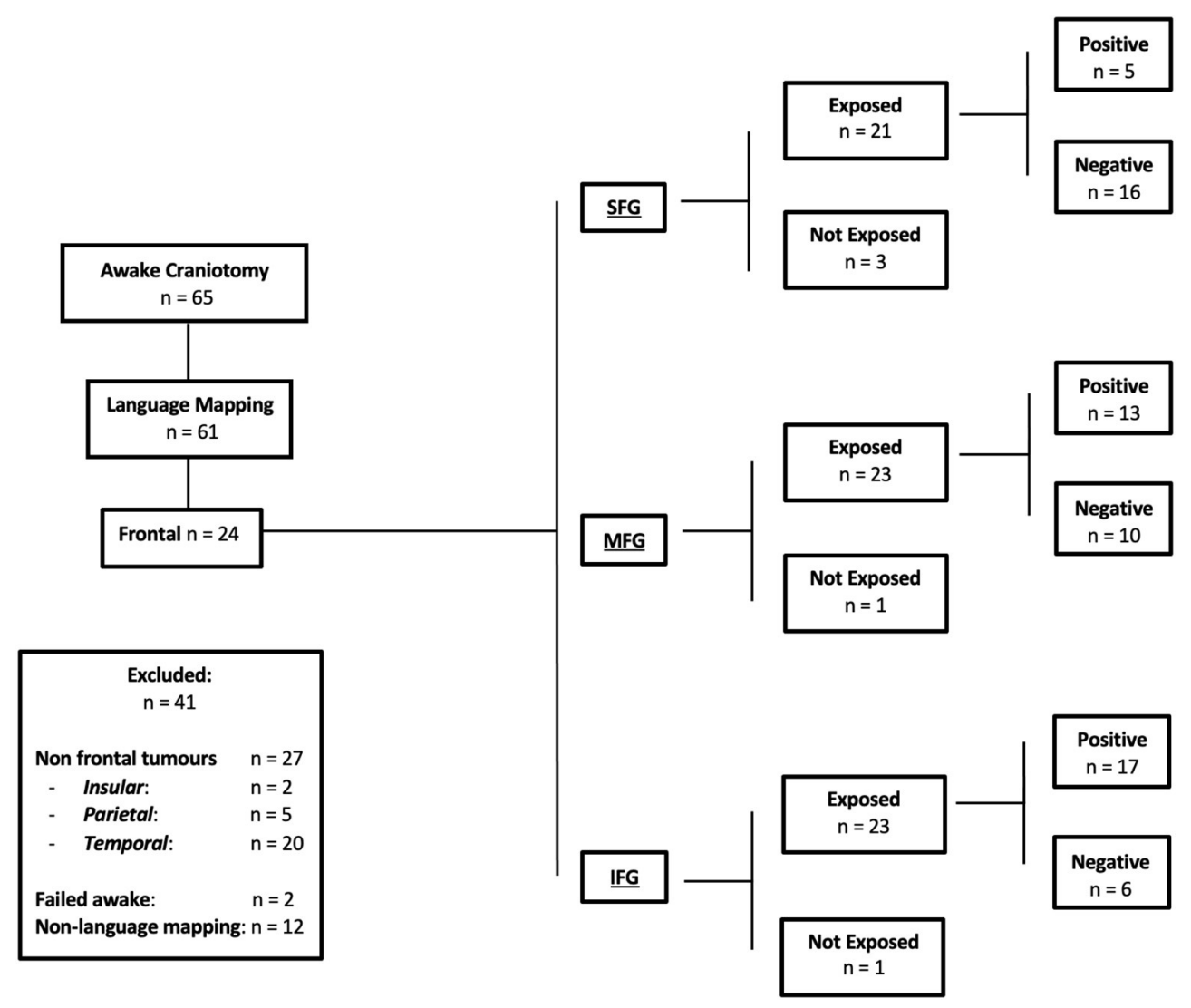

FIGURE 1 | Flow chart of the present study, detailing the inclusion and exclusion criteria. Positive responses indicate language errors elicited by direct electric stimulation. Negative responses indicate no language errors elicited by direct electric stimulation. Exposure indicates the specific frontal gyrus exposed during craniotomy.

Dutch Linguistic Intra-operative Protocol (31) and the Verb and Noun Test for Peri-Operative Testing (32).

Positive and negative responses were recorded, indicating the presence and absence of language errors elicited by DES respectively. After a speech arrest was identified and the threshold was established, the entire exposed cortical area of the frontal lobe was mapped with object naming and action naming in both present and past tenses. At the subcortical level, repetition tasks were used for mapping the arcuate fasciculus (AF), sentence completion for the fronto-aslant tract (FAT), and semantic odd-one-out and object naming for the inferior frontooccipital fasciculus (IFOF). The positive responses-two out of three attempts-with induced speech deficit in the absence of after-discharges on electrocorticography were documented with intraoperative pictures.

\section{Navigated Transcranial Magnetic Stimulation}

Preoperative mapping with nTMS has been used at our center since 2016 as an adjunct to intraoperative DES mapping. Whenever available, data from nTMS were included in the analysis.
Language mapping was performed following resting motor threshold determination (33). A set series of pre-designed images were presented to the patient for baseline assessment in two consecutive rounds: object naming, action naming in the present tense, and action naming in the past tense (32). The inter-picture interval was set to $2,500 \mathrm{~ms}$, and the display time (DT) varied between 500 and 1,000 ms, which was dependent on a patient's ability. Images that were incorrectly described or with hesitation were excluded from the final exam. An offline analysis was performed, comparing the stimulation assessment to the baseline assessment and identifying any changes in language function during the exam. Language errors were classified into distinct categories (hesitation, expressive, semantic, anomia, arrest, other, and no errors).

The intraoperative pictures of positive stimulation sites collected during intraoperative mapping were included in the preoperative MRI studies (T1-weighted images after gadolinium injection) by comparing the anatomical landmarks (i.e., sulci and gyri) of the single pictures with the axial brain volumetric images and reformatted sagittal/coronal images (33). It was therefore possible to correlate the positive intraoperative sites with nTMS mapping, allowing for the calculation of TMS 
specificity, sensitivity, and positive and negative predictive values (PPV and NPV, respectively).

\section{Statistical Analysis}

STATA 13.0 software was used for statistical analysis. Regression techniques were performed to compare the language outcomesscreening test for acquired language disorder (SST) and its receptive and expressive subdivisions-with the number of gyri and the main subcortical areas infiltrated by the tumor. A $p$-value $<0.05$ was considered as significant.

\section{RESULTS}

Sixty-five awake craniotomies were performed between 2012 and 2020. There were 24 (36.9\%) frontal tumors that constitute the object of the current study. The demographics showed an even distribution between males and females, with a mean age of $47 \pm 13.8$ years old (standard deviation). The majority of the included patients had left-sided tumors $(22,91.6 \%)$. Meanwhile, $19(79.1 \%)$ patients presented with a new-onset seizure; two (8.3\%) had a motor deficit, and two $(8.3 \%)$ presented with a language deficit. The vast majority of patients $(20,83.4 \%)$ presented with a performance status of 0 . High-grade gliomas were prevalent in this series [71\% World Health Organization (WHO) grade III and IV]. Isocitrate dehydrogenase was positive in $10(41.6 \%)$ tumors, whereas $1 \mathrm{p} / 19 \mathrm{q}$ co-deletion was present in nine (37.5\%) oligodendrogliomas. Figure 1 and Table 1 summarize the patients' characteristics.

\section{Preoperative Language Assessment}

The average preoperative SST was 18.9/20. The average receptive language skill score was $8.1 / 9$, and the average expressive language skill score was 10.7/11 (seven patients were excluded due to incomplete assessments). From the SST, the most frequent receptive errors were auditory semantic differentiation and the ability to fully comprehend a paragraph-level narrative. Expressive errors were rare, but when present, the most frequent error was the ability to provide word definitions. The SST does not measure phonemic ability, but no patient displayed any marked phonemic errors in conversation preoperatively (Table 2).

\section{Preoperative nTMS Assessment}

nTMS was performed in $14(58.3 \%)$ patients with frontal tumors (1,844 stimulations distributed across the gyri as follows: SFG-172, MFG-428, IFG-1,244), and positive responses were elicited in 12 patients (85.7\%), with a total of 42 positive responses: three responses in the SFG (one hesitation and two anomic errors); seven responses in the MFG (five hesitations and two phonemic errors); 32 responses in the IFG (24 hesitations, six phonemic, and two semantic errors). This shows a preferential distribution of the stimulations in the IFG, particularly given the likelihood of positive responses in the area of the frontal operculum.

Overall, the nTMS had a PPV of $31.0 \%$, a NPV of $97.8 \%$, sensitivity of $45.7 \%$, and specificity of $98.3 \%$. When the gyri were compared, the MFG had the highest PPV (43.0\% vs. IFG-31.25\%
TABLE 1 | Demographics of the included subjects along with the classification of tumors based on histology, grade, and biomarkers.

\section{Subject demographics and tumor classification}

\section{Sex}

Female

$12(50 \%)$

Male

$12(50 \%)$

Age group

18-24

25-34

$35-44$

$45-54$

$55-64$

$1(4.1 \%)$

$5(20.8 \%)$

$5(20.8 \%)$

$5(20.8 \%)$

$6(25 \%)$

$2(8.3 \%)$

Presentation

Motor deficit

$2(8.3 \%)$

Language deficit

2 (8.3\%)

Cognitive deficit

1 (4.2\%)

Seizure

19 (79.2\%)

Tumor laterality

Right

2 (8.3\%)

Left

22 (91.6\%)

Histology

Anaplastic Astrocytoma

Anaplastic Oligodendroglioma

Diffuse Astrocytoma

Glioblastoma Multiforme

Glioneuronal Tumor

High Grade Glioma

Low Grade Glioma

Oligodendroglioma

WHO grading

I

II

II

III

IV

Tumor marker

IDH

Positive

(41.6\%)

Negative

$7(29.1 \%)$

Mutant

6 (25\%)

Wildtype

$1(4.2 \%)$

1p/19q

Co deletion

$9(37.5 \%)$

No deletion

2 (8.3\%)

19q deletion

1 (4.2\%)

N/A

12 (50\%)

and SFG-0\%), NPV (98.3\% vs. IFG-97.8\% and SFG-95.9\%), sensitivity $(50.0 \%$ vs. IFG-47.1\%, and SFG-30.0\%), and specificity (99.0\% vs. IFG-98.2\% and SFG-98.1\%) (Table 2, Figure 2, and Supplementary Video 1). 
TABLE 2 | A comprehensive table detailing the navigated transcranial magnetic simulation language errors, intraoperative stimulation positive responses, a comparison of language pathway error, Sheffield Aphasia Screening Test (SST) for Acquired Language Disorder assessments pre- and postoperatively, changes in SST score, and a comprehensive speech and language therapy pre-, intra-, and postoperative assessment.

\begin{tabular}{|c|c|c|c|c|c|c|c|c|}
\hline \multirow[t]{2}{*}{ ID } & \multirow{2}{*}{$\begin{array}{l}\text { nTMS Language } \\
\text { Error }\end{array}$} & \multirow{2}{*}{$\begin{array}{l}\text { Intra-operative } \\
\text { mapping } \\
\text { response }\end{array}$} & \multicolumn{3}{|c|}{ Sheffield screening test (SST) } & \multicolumn{3}{|c|}{ Speech and language therapist (SLT) assessment } \\
\hline & & & Preoperative & Postoperative & $\begin{array}{l}\text { Difference in } \\
\text { scores }\end{array}$ & Preoperative & Intraoperative & Postoperative \\
\hline 1 & $\begin{array}{l}\text { SFG: None } \\
\text { MFG: Stutter (1) } \\
\text { IFG: Hesitation (3) }\end{array}$ & SFG-MFG & $\begin{array}{l}\text { Total: } \\
\text { Spanish: 18/20 } \\
\text { English: 13/20 }\end{array}$ & $\begin{array}{l}\text { None } \\
\frac{2 \text { years post op: }}{12 / 20}\end{array}$ & Total: -6 & $\begin{array}{l}\text { Mild receptive and expressive } \\
\text { dysphasia } \\
\text { Semantic errors in Spanish } \\
\text { Action and object } \\
\text { naming difficulties }\end{array}$ & $\begin{array}{l}\text { No language errors in English } \\
\text { or Spanish }\end{array}$ & $\begin{array}{l}\text { Mild receptive and expressive } \\
\text { dysphasia } \\
\text { Increase in word finding difficulties } \\
\text { compared to pre op (in } \\
\text { both languages) }\end{array}$ \\
\hline 2 & $\begin{array}{l}\text { SFG: None } \\
\text { MFG: Word finding } \\
\text { difficulty (1) } \\
\text { IFG: None }\end{array}$ & SFG-MFG & $\begin{array}{l}\text { Total: } 15 / 20 \\
\text { Receptive 6/9 } \\
\text { Expressive 9/11 }\end{array}$ & $\begin{array}{l}\text { Total: } \\
\text { 9/20 } \\
\text { Receptive 4/9 } \\
\text { Expressive 5/11 }\end{array}$ & $\begin{array}{l}\text { Total: }-6 \\
\text { Receptive }-2 \\
\text { Expressive }-4\end{array}$ & $\begin{array}{l}\text { No obvious dysphasia } \\
\text { Difficulties more likely to be due } \\
\text { to English as additional } \\
\text { Action and object } \\
\text { naming difficulties }\end{array}$ & $\begin{array}{l}\text { Semantic errors, hesitation in } \\
\text { Farsi for object naming. } \\
\text { End of awake period: difficulty } \\
\text { with object naming in both } \\
\text { languages } \\
\text { More difficulty in first language } \\
\text { (Farsi) than in English }\end{array}$ & $\begin{array}{l}\text { Moderate dysphasia in English } \\
\text { Word finding difficulties (semantic } \\
\text { errors) and perseveration in English ( } 5 \\
\text { days post op) }\end{array}$ \\
\hline 3 & $\begin{array}{l}\text { SFG: None } \\
\text { MFG: None } \\
\text { IFG: Hesitation, } \\
\text { Apraxia, Semantic } \\
\text { Anomia (13) }\end{array}$ & IFG-MFG & Total: 20/20 & $\begin{array}{l}2 \text { days post op: unable } \\
\text { to participate } \\
5 \text { days post op: } \\
\text { Total: } 14 / 20 \\
\text { Receptive } 8 / 9 \\
\text { Expressive } 6 / 11\end{array}$ & $\begin{array}{l}\text { Total: }-6 \\
\text { Receptive }-1 \\
\text { Expressive }-5\end{array}$ & $\begin{array}{l}\text { No overt dysphasia } \\
\text { in conversation }\end{array}$ & $\begin{array}{l}\text { Hesitation and semantic error } \\
\text { (object naming, action naming) } \\
\text { with } 2 \text { perseveration errors in } \\
\text { object naming }\end{array}$ & $\begin{array}{l}\text { Mild/moderate dysphasia } \\
\text { Difficulty with complex reading tasks } \\
\text { Hesitancy } \\
\text { Word finding difficulty }\end{array}$ \\
\hline 4 & $\begin{array}{l}\text { SFG: None } \\
\text { MFG: None } \\
\text { IFG: None }\end{array}$ & SFG-MFG & $\begin{array}{l}\text { Total: 19/20 } \\
\text { Receptive 8/9 } \\
\text { Expressive 11/11 }\end{array}$ & $\begin{array}{l}\text { Total: } 2 / 20 \\
\text { Receptive } 2 / 9 \text {, test } \\
\text { abandoned as } \\
\text { too difficult }\end{array}$ & $\begin{array}{l}\text { Total: }-17 \\
\text { Receptive }-6\end{array}$ & $\begin{array}{l}\text { No communication and } \\
\text { language dysfunction }\end{array}$ & $\begin{array}{l}\text { Semantic, phonemic, reading, } \\
\text { and fluency errors }\end{array}$ & $\begin{array}{l}\text { Moderate expressive and receptive } \\
\text { dysphasia } \\
\text { Fluency errors and difficulties with } \\
\text { semantic tasks } \\
\text { Spontaneous speech notably easier } \\
\text { than when asked direct questions }\end{array}$ \\
\hline 5 & $\begin{array}{l}\text { SFG: None } \\
\text { MFG: None } \\
\text { IFG: Not performed }\end{array}$ & SFG-MFG & $\begin{array}{l}\text { Total: 19/20 } \\
\text { Receptive 8/9 } \\
\text { Expressive 11/11 }\end{array}$ & $\begin{array}{l}\text { Unable to complete } \\
\text { SST } \\
\frac{7 \text { days post op: Total: }}{13 / 20} \\
\text { Receptive } 8 / 9 \\
\text { Expressive } 5 / 11\end{array}$ & $\begin{array}{l}\text { Total: }-6 \\
\text { Receptive } 0 \\
\text { Expressive -6 }\end{array}$ & Semantic errors & $\begin{array}{l}\text { Semantic errors } \\
\text { SMA-like syndrome } \\
\text { No automatic speech, } \\
\text { counting errors }\end{array}$ & $\begin{array}{l}\text { Semantic and phonemic errors } \\
\text { Moderate expressive aphasia }\end{array}$ \\
\hline 6 & Not performed & SFG-MFG & Total: 20/20 & Total: $18 / 20$ & Total: -2 & $\begin{array}{l}\text { Difficulty following complex } \\
\text { commands } \\
\text { Phonemic errors in conversation } \\
\text { reported by family but not seen } \\
\text { in clinic }\end{array}$ & $\begin{array}{l}\text { No difficulties with repetition, } \\
\text { object or verb naming }\end{array}$ & $\begin{array}{l}5 \text { days post op: mild receptive and } \\
\text { severe expressive dysphasia with } \\
\text { likely overlay of verbal dyspraxia } \\
1 \text { month post op: mild expressive } \\
\text { dysphasia } \\
\text { Semantic word finding difficulties in } \\
\text { conversation and/or difficulties with } \\
\text { grammatical structure }\end{array}$ \\
\hline
\end{tabular}




\begin{tabular}{|c|c|c|c|c|c|c|c|c|}
\hline \multirow[t]{2}{*}{ ID } & \multirow{2}{*}{$\begin{array}{l}\text { nTMS Language } \\
\text { Error }\end{array}$} & \multirow{2}{*}{$\begin{array}{l}\text { Intra-operative } \\
\text { mapping } \\
\text { response }\end{array}$} & \multicolumn{3}{|c|}{ Sheffield screening test (SST) } & \multicolumn{3}{|c|}{ Speech and language therapist (SLT) assessment } \\
\hline & & & Preoperative & Postoperative & $\begin{array}{l}\text { Difference in } \\
\text { scores }\end{array}$ & Preoperative & Intraoperative & Postoperative \\
\hline 7 & $\begin{array}{l}\text { SFG: Hesitation (1) } \\
\text { MFG: Hesitation (1) } \\
\text { IFG: Word formation (1) }\end{array}$ & SFG-MFG & $\begin{array}{l}\text { Total: 19/20 } \\
\text { Receptive 8/9 } \\
\text { Expressive 11/11 }\end{array}$ & $\begin{array}{l}5 \text { days post op unable } \\
\text { to complete: severe } \\
\text { expressive dysphasia, } \\
\text { SMA initiation } \\
\text { difficulties. } \\
7 \text { days post op: } \\
\text { Total: } 13 / 20 \\
\text { Receptive } 7 / 9 \\
\text { Expressive } 6 / 11\end{array}$ & $\begin{array}{l}\text { Total: }-6 \\
\text { Receptive }-1 \\
\text { Expressive }-5\end{array}$ & $\begin{array}{l}\text { No overt dysphasia } \\
\text { in conversation. }\end{array}$ & $\begin{array}{l}\text { Speech arrest } \\
2 \text { semantic errors } \\
1 \text { hesitation error (all in verb } \\
\text { naming) } \\
2 \text { sentence completion errors }\end{array}$ & $\begin{array}{l}\text { Mild receptive with moderate } \\
\text { expressive dysphasia } \\
\text { Semantic difficulties with additional } \\
\text { difficulties initiating speech }\end{array}$ \\
\hline 8 & $\begin{array}{l}\text { SFG: None } \\
\text { MFG: None } \\
\text { IFG: Hesitation (6) }\end{array}$ & IFG-MFG & $\begin{array}{l}\text { Total: 18/20 } \\
\text { Receptive 8/9 } \\
\text { Expressive 10/11 }\end{array}$ & $\begin{array}{l}\text { Total: 18/20 } \\
\text { Receptive 8/9 } \\
\text { Expressive 10/11 }\end{array}$ & $\begin{array}{l}\text { Total: } 0 \\
\text { Receptive } 0 \\
\text { Expressive } 0\end{array}$ & $\begin{array}{l}\text { No overt dysphasia in } \\
\text { conversation but semantic } \\
\text { difficulties in testing. }\end{array}$ & $\begin{array}{l}\text { Semantic, hesitation, } \\
\text { fluency errors. }\end{array}$ & $\begin{array}{l}\text { Mild dysphasia - mild word finding } \\
\text { difficulties in conversation - using } \\
\text { circumlocution to good effect }\end{array}$ \\
\hline 9 & $\begin{array}{l}\text { SFG: Anomia (2) } \\
\text { MFG: Hesitation (2) } \\
\text { IFG: None }\end{array}$ & SFG-MFG & $\begin{array}{l}\text { Total: } 18 / 20 \\
\text { Receptive } 7 / 9 \\
\text { Expressive 11/11 }\end{array}$ & $\begin{array}{l}\text { Total: } 9 / 20 \\
\text { Receptive } 2 / 9 \\
\text { Expressive } 7 / 11 \\
\text { (cognitive overlay) }\end{array}$ & $\begin{array}{l}\text { Total: }-9 \\
\text { Receptive }-5 \\
\text { Expressive }-4\end{array}$ & $\begin{array}{l}\text { No overt difficulties, occasional } \\
\text { hesitations } \\
\text { Semantic errors }\end{array}$ & $\begin{array}{l}\text { Word finding difficulties in } \\
\text { conversation and object naming }\end{array}$ & $\begin{array}{l}\text { Mild dysarthria, moderate dysphasia } \\
\text { with phonemic errors in conversation } \\
\text { Cognitive communication difficulties }\end{array}$ \\
\hline 10 & $\begin{array}{l}\text { SFG: None } \\
\text { MFG: None } \\
\text { IFG: Hesitation (6) }\end{array}$ & IFG-MFG & Total: 20/20 & $\begin{array}{l}\text { Total: } 14 / 20 \\
\text { Receptive } 9 / 9 \\
\text { Expressive } 5 / 11\end{array}$ & $\begin{array}{l}\text { Total: }-6 \\
\text { Receptive } 0 \\
\text { Expressive }-6\end{array}$ & No overt dysphasia & 2 phonemic errors in mapping & $\begin{array}{l}\text { Mild to moderate expressive } \\
\text { dysphasia } \\
\text { Motor planning difficulties }\end{array}$ \\
\hline 11 & $\begin{array}{l}\text { SFG: None } \\
\text { MFG: Hesitation (1) } \\
\text { IFG: None }\end{array}$ & IFG-MFG & $\begin{array}{l}\text { Total: } 17 / 20 \\
\text { Receptive 6/9 } \\
\text { Expressive 11/11 }\end{array}$ & Total: 20/20 & $\begin{array}{l}\text { Total: }+3 \\
\text { Receptive }+3 \\
\text { Expressive } 0\end{array}$ & $\begin{array}{l}\text { No communication difficulties } \\
\text { in conversation }\end{array}$ & $\begin{array}{l}\text { Imprecise articulation/dysarthric } \\
\text { errors in mapping (2) and in } \\
\text { cortical resection }\end{array}$ & No obvious dysphasia \\
\hline 12 & Not performed & None & Not performed & Not performed & $\begin{array}{l}\text { Unable to } \\
\text { determine }\end{array}$ & $\begin{array}{l}\text { No obvious dysphasia in first } \\
\text { language (Polish) }\end{array}$ & $\begin{array}{l}\text { Perseveration and word finding } \\
\text { difficulties during resection }\end{array}$ & No data available \\
\hline 13 & Not performed & IFG-MFG & $\begin{array}{l}\text { Total: 19/20 } \\
\text { Receptive 8/9 } \\
\text { Expressive 11/11 }\end{array}$ & $\begin{array}{l}\text { Total: } 14 / 20 \\
\text { Receptive 6/9 } \\
\text { Expressive 8/11 }\end{array}$ & $\begin{array}{l}\text { Total: }-5 \\
\text { Receptive }-2 \\
\text { Expressive }-3\end{array}$ & $\begin{array}{l}\text { Higher level word } \\
\text { finding difficulties }\end{array}$ & $\begin{array}{l}4 \text { phonemic errors in mapping, } \\
1 x \text { phonemic error and } 1 x \\
\text { hesitation in resection }\end{array}$ & $\begin{array}{l}\text { Mild receptive and expressive } \\
\text { dysphasia Word finding difficulties } \\
\text { more evident in testing than } \\
\text { in conversation }\end{array}$ \\
\hline 14 & $\begin{array}{l}\text { SFG: None } \\
\text { MFG: Hesitation (1) } \\
\text { IFG: Hesitation, } \\
\text { Anomia (2) }\end{array}$ & IFG-MFG & $\begin{array}{l}\text { Total: 20/20 } \\
\text { Receptive 9/9 } \\
\text { Expressive 11/11 }\end{array}$ & $\begin{array}{l}\text { Total: } 15 / 20 \\
\text { Receptive } 7 / 9 \\
\text { Expressive 8/11 }\end{array}$ & $\begin{array}{l}\text { Total: }-5 \\
\text { Receptive }-2 \\
\text { Expressive }-3\end{array}$ & Initial difficulty in recalling details & $\begin{array}{l}\text { Phonemic errors noted in } \\
\text { mapping ( } 2 \text { separate areas) } \\
\text { Self-correcting phonemic errors } \\
\text { in conversation during resection } \\
1 \text { phonemic error at final testing } \\
\text { at the end of resection }\end{array}$ & $\begin{array}{l}\text { Mild receptive and expressive } \\
\text { dysphasia } \\
\text { Improvements noted in verbal } \\
\text { sequencing compared to immediately } \\
\text { after previous surgery }\end{array}$ \\
\hline 15 & $\begin{array}{l}\text { SFG: None } \\
\text { MFG: None } \\
\text { IFG: Hesitation, } \\
\text { Dysarthria, Semantic (4) }\end{array}$ & IFG-MFG & Total: 20/20 & $\begin{array}{l}\text { Total: } 18 / 20 \\
\text { Receptive } 8 / 9 \\
\text { Expressive 10/11 } \\
\text { (phonemic errors not } \\
\text { significant to impact on } \\
\text { score) }\end{array}$ & $\begin{array}{l}\text { Total: }-2 \\
\text { Receptive }-1 \\
\text { Expressive-1 }\end{array}$ & No communication difficulties & $\begin{array}{l}\text { Action naming, } 1 \text { clear speech } \\
\text { arrest in lead up phrase } \\
\text { Object naming, phonemic } \\
\text { difficulty during mapping, } \\
\text { resection of arcuate fasciculus } \\
\text { Slurred speech at the end } \\
\text { of resection }\end{array}$ & $\begin{array}{l}\text { Mild word finding difficulties with } \\
\text { lower frequency nouns } \\
\text { Mildly reduced associated naming } \\
\text { Mild-moderate verbal apraxia, mild } \\
\text { dysarthria and mild } \\
\text { expressive dysphasia }\end{array}$ \\
\hline
\end{tabular}

Preoperative 
TABLE 2 | Continued

\begin{tabular}{|c|c|c|c|c|c|c|c|c|}
\hline \multirow[t]{2}{*}{ ID } & \multirow{2}{*}{$\begin{array}{l}\text { nTMS Language } \\
\text { Error }\end{array}$} & \multirow{2}{*}{$\begin{array}{l}\text { Intra-operative } \\
\text { mapping } \\
\text { response }\end{array}$} & \multicolumn{3}{|c|}{ Sheffield screening test (SST) } & \multicolumn{3}{|c|}{ Speech and language therapist (SLT) assessment } \\
\hline & & & Preoperative & Postoperative & $\begin{array}{l}\text { Difference in } \\
\text { scores }\end{array}$ & Preoperative & Intraoperative & Postoperative \\
\hline 16 & Not performed & None & Not performed & Not performed & $\begin{array}{l}\text { Unable to } \\
\text { determine }\end{array}$ & $\begin{array}{l}\text { No communication } \\
\text { difficulties reported }\end{array}$ & No data available & $\begin{array}{l}\text { Mild receptive and moderate-severe } \\
\text { expressive dysphasia } \\
\text { Unable to speak in phrases or } \\
\text { sentences, occasional word, } \\
\text { using Yes/No }\end{array}$ \\
\hline 17 & Not performed & None & Not performed & Not performed & $\begin{array}{l}\text { Unable to } \\
\text { determine }\end{array}$ & $\begin{array}{l}\text { All normal except planning } \\
\text { (constructing sentences from } \\
\text { given words) } \\
\text { Mildly impaired in efficiency, } \\
\text { auditory memory, auditory } \\
\text { comprehension and numeracy }\end{array}$ & $\begin{array}{l}\text { Prompting for biological } \\
\text { information, one-word answers, } \\
\text { Counting: perseveration at } \\
\text { number } 7 \\
\text { Visual and semantic errors on } \\
\text { picture naming } \\
\text { Speech arrest }\end{array}$ & $\begin{array}{l}2 \text { days post op: moderate receptive } \\
\text { and expressive dysphasia } \\
\text { Semantic and phonemic errors } \\
\text { Speech slow and effortful } \\
5 \text { days post op: Mild/moderate } \\
\text { dysphasia but still with phonemic and } \\
\text { semantic errors }\end{array}$ \\
\hline 18 & Not performed & None & Not performed & Not performed & $\begin{array}{l}\text { Unable to } \\
\text { determine }\end{array}$ & $\begin{array}{l}\text { No overt dysphasia } \\
\text { Mild difficulties with planning for } \\
\text { sentence construction and in } \\
\text { planning for sequencing on } \\
\text { Mount Wilga higher level } \\
\text { language tasks } \\
\text { Some word finding difficulties } \\
\text { previous to taking steroids }\end{array}$ & $\begin{array}{l}\text { Minor visual and semantic errors } \\
\text { Speech arrest very obvious } \\
\text { during stimulation when counting } \\
1-10 \text { during first half of testing } \\
\text { No obvious dysphasia in } \\
\text { intra-operative testing. } \\
\text { At end of testing, able to name } \\
\text { single object pictures, describe } \\
\text { pictures, repeat words and } \\
\text { participate in conversation }\end{array}$ & $\begin{array}{l}\text { No obvious dysphasia in conversation } \\
\text { or Brisbane Language screen }\end{array}$ \\
\hline 19 & Not performed & None & Not performed & Not performed & $\begin{array}{l}\text { Unable to } \\
\text { determine }\end{array}$ & $\begin{array}{l}\text { No difficulties communicating in } \\
\text { conversation } \\
\text { Mild higher-level language } \\
\text { difficulties in Mount Wilga tests } \\
\text { ( } 7 / 10 \text { in auditory comprehension } \\
\text { and recall questions, difficulty } \\
\text { with jumbled sentences task) } \\
\text { No difficulty with planning tasks. }\end{array}$ & No communication errors & $\begin{array}{l}\text { Mild dysphasia } \\
\text { Word finding difficulties in } \\
\text { conversation (phonemic and } \\
\text { semantic) and difficulties organizing } \\
\text { sentences within a narrative }\end{array}$ \\
\hline 20 & $\begin{array}{l}\text { SFG: None } \\
\text { MFG: None } \\
\text { IFG: None }\end{array}$ & SFG-MFG & Total: 20/20 & $\begin{array}{l}\text { Post op: } \\
\text { Total: 0/20 } \\
\text { Post op week 1: } \\
\text { Total: } 8 / 20 \\
\text { Post op week 2: } \\
\text { Total: } 14 / 20 \\
\text { Receptive } 9 / 9 \\
\text { Expressive } 5 / 11\end{array}$ & $\begin{array}{l}\text { Total: }-6 \\
\text { Receptive } 0 \\
\text { Expressive }-6\end{array}$ & No difficulties in communication & $\begin{array}{l}\text { No issues with naming objects } \\
\text { and actions } \\
\text { Possible hesitation with lower } \\
\text { frequency items } \\
\text { Later stages of resection, able to } \\
\text { name intermittently, preservation } \\
\text { and mild semantic errors noted } \\
\text { At the end of resection, unable to } \\
\text { name or repeat or count to } 10\end{array}$ & $\begin{array}{l}\text { Severe expressive and receptive } \\
\text { dysphasia } \\
\text { Non-verbal post-op } \\
\text { Overlay of difficulties initiating speech } \\
\text { - at times these severely impact on } \\
\text { patient's ability to make } \\
\text { self understood }\end{array}$ \\
\hline
\end{tabular}


TABLE 2 | Continued

\begin{tabular}{|c|c|c|c|c|c|c|c|c|}
\hline \multirow[t]{2}{*}{ ID } & \multirow{2}{*}{$\begin{array}{l}\text { nTMS Language } \\
\text { Error }\end{array}$} & \multirow{2}{*}{$\begin{array}{l}\text { Intra-operative } \\
\text { mapping } \\
\text { response }\end{array}$} & \multicolumn{3}{|c|}{ Sheffield screening test (SST) } & \multicolumn{3}{|c|}{ Speech and language therapist (SLT) assessment } \\
\hline & & & Preoperative & Postoperative & $\begin{array}{l}\text { Difference in } \\
\text { scores }\end{array}$ & Preoperative & Intraoperative & Postoperative \\
\hline 21 & Not performed & SFG-MFG & $\begin{array}{l}\text { Total: } 19 / 20 \\
\text { Receptive } 8 / 9 \\
\text { Expressive 11/11 }\end{array}$ & $\begin{array}{l}\text { Total: } 20 / 20 \\
\text { Receptive } 9 / 9 \\
\text { Expressive } 11 / 11\end{array}$ & $\begin{array}{l}\text { Total: }+1 \\
\text { Receptive }+1 \\
\text { Expressive } 0\end{array}$ & No communication difficulties & $\begin{array}{l}\text { No communication errors } \\
\text { but drowsy }\end{array}$ & $\begin{array}{l}\text { No obvious dysphasia or difficulties in } \\
\text { short conversation }\end{array}$ \\
\hline 22 & Not performed & None & Not performed & Not performed & $\begin{array}{l}\text { Unable to } \\
\text { determine }\end{array}$ & $\begin{array}{l}\text { Mild difficulty with written } \\
\text { calculation and planning } \\
\text { sentence construction }\end{array}$ & $\begin{array}{l}\text { Periods of motor speech and } \\
\text { naming deficits during mapping } \\
\text { and surgery. } \\
\text { At end of SLT assessment: } \\
\text {-Decreased spontaneous verbal } \\
\text { output -Producing automatics } \\
\text { and single words/short phrases } \\
\text { to sentence closure tasks }\end{array}$ & $\begin{array}{l}2 \text { days post op: severe expressive } \\
\text { dysphasia } \\
5 \text { days post op: mild receptive with } \\
\text { moderate expressive dysphasia } \\
\text { Dyspraxia of speech, comprehending } \\
\text { complex info during conversations. }\end{array}$ \\
\hline 23 & $\begin{array}{l}\text { SFG: None } \\
\text { MFG: None } \\
\text { IFG: Hesitation, } \\
\text { Anomia (2) }\end{array}$ & IFG-MFG & $\begin{array}{l}\text { Total: } 18 / 20 \\
\text { Receptive 8/9 } \\
\text { Expressive 10/11 }\end{array}$ & $\begin{array}{l}\text { Total: } 16 / 20 \\
\text { Receptive } 7 / 9 \\
\text { Expressive 9/11 }\end{array}$ & $\begin{array}{l}\text { Total: }-2 \\
\text { Receptive }-1 \\
\text { Expressive-1 }\end{array}$ & $\begin{array}{l}\text { Some higher-level language } \\
\text { difficulties apparent }\end{array}$ & $\begin{array}{l}\text { Minor difficulty with repetition } \\
\text { during subcortical mapping } \\
\text { Intermittent repetition, naming, } \\
\text { and spontaneous speech during } \\
\text { resection } \\
\text { No difficulties with spontaneous } \\
\text { speech at the end of surgery; } \\
\text { able to answer direct questions, } \\
\text { name high frequency objects } \\
\text { and } 9 / 10 \text { on repetition tasks } \\
\text { but fatigued }\end{array}$ & $\begin{array}{l}\text { Mild dysphasia and higher level } \\
\text { language difficulties likely linked to } \\
\text { ability to retain and } \\
\text { organize information }\end{array}$ \\
\hline 24 & $\begin{array}{l}\text { SFG: None } \\
\text { MFG: None } \\
\begin{array}{l}\text { IFG: Language } \\
\text { reversion to French (1) }\end{array}\end{array}$ & $\begin{array}{l}\text { SFG-MFG } \\
\text { IFG-MFG }\end{array}$ & Total: 20/20 & $\begin{array}{l}\text { Total: } 13 / 20 \\
\text { Receptive 6/9 } \\
\text { Expressive } 7 / 11\end{array}$ & $\begin{array}{l}\text { Total: }-7 \\
\text { Receptive }-3 \\
\text { Expressive }-4\end{array}$ & $\begin{array}{l}\text { Mild to moderate impairment in } \\
\text { verbal explanation. } \\
\text { Moderate impairment in } \\
\text { sentence construction and } \\
\text { understanding inferential } \\
\text { information, this is likely akin to } \\
\text { mild dysphasia though EAL } \\
\text { (English as an } \\
\text { Additional Language) }\end{array}$ & $\begin{array}{l}\text { Semantic errors in initial } \\
\text { mapping, too drowsy to } \\
\text { comment on phonemic errors }\end{array}$ & $\begin{array}{l}\text { Mild/moderate receptive and } \\
\text { expressive dysphasia } \\
\text { Able to answer basic questions in } \\
\text { conversation but difficulty with longer } \\
\text { explanations: likely combination of } \\
\text { cognitive communication difficulty } \\
\text { and dysphasia }\end{array}$ \\
\hline
\end{tabular}


A

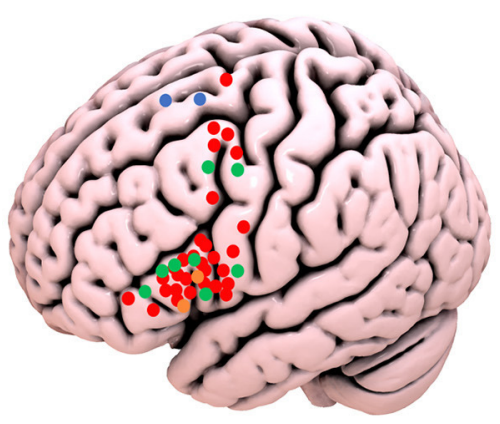

C

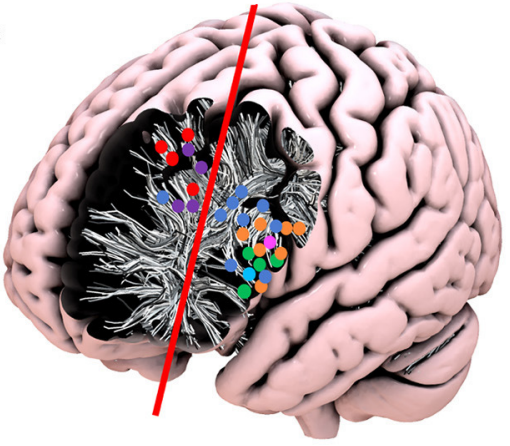

B

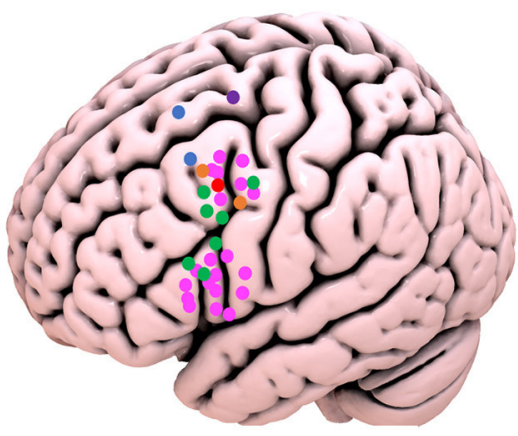

Hesitation

Phonemic

Anomic

Semantic

Fluency

Speech Arrest

FIGURE 2 | Schematic representation of the positive navigated transcranial magnetic simulation responses, intraoperative cortical responses, and positive subcortical responses according to the different language errors. (A) Positive TMS responses. (B) Positive cortical intraoperative responses. (C) Positive subcortical intraoperative responses.

A preoperative structural connectome analysis performed in three patients with positive responses in the area 55b showed the strongest connectivity of this area not only with the primary motor cortex but as well as with the supplementary motor area, inferior frontal gyrus, and anterior aspect of the MFG (Figure 3). Positive nTMS mapping in the inferior frontal gyrus was related with a better preoperative overall SST score $(p=0.017)$ due to a better receptive component $(p=0.001)$. Positive nTMS mapping for the posterior MFG/area $55 \mathrm{~b}$ was related with a worse receptive preoperative component of the SST $(p=0.031)$, but with no expression in the overall score $(p=0.059)$ (Table 3).

\section{Intraoperative Speech Errors}

The superior frontal gyrus (SFG) was exposed in 21 (87.5\%), the middle frontal gyrus (MFG) in 23 (95.8\%), and the inferior frontal gyrus (IFG) in $23(95.8 \%)$ craniotomies. A total of 33 cortical positive responses for language were recorded. Two (6.0\%) responses were recorded in the SFG, $15(45.5 \%)$ in the MFG, and $16(48.5 \%)$ in the IFG. Of relevance is the fact that all positive responses recorded in the MFG were demonstrated in area 55b. The following responses were identified: speech arrest in 20 patients (13 in IFG; seven in area 55b), hesitation in one patient (area 55b), decreased fluency in one patient (SFG), phonemic errors in seven patients (four in area 55b and three in IFG), semantic paraphasias in two patients (area 55b), and anomia in two patients (one in MFG and one in SFG). At the subcortical level, 29 positive responses were identified and divided into two main areas: 21 patients in the deep IFG-MFG area and eight patients in the deep MFG-SFG area. These positive responses were divided as follows: repetition disturbance in one patient (deep IFG), speech arrest in one patient (deep IFG), hesitation in four patients (deep MFG-SFG), decreased fluency in three patients (deep SFG), phonemic errors in four patients (deep IFG-MFG), semantic paraphasias in seven patients (deep IFG-MFG), and nine anomias (eight in deep IFG-MFG and one in deep MFG-SFG) (Figure 2).

\section{Postoperative Language Assessment}

Twenty (83.3\%) patients had a transient deterioration of their language function after surgery (mean postoperative SST $=$ $14.67 \pm 0.76)$. Both the expressive $(-2.875 \pm 0.55)$ and the receptive $(-1.36 \pm 0.37)$ components of the SST deteriorated, with a statistically significant greater deterioration of the expressive component $(p=0.03)$. The single involvement of a particular gyrus (including area 55b) was not related per se with significant changes in language outcomes. The number of gyri with documented intraoperative positive language mapping was correlated with language outcomes: an increased number of gyri involvement was related with a better preoperative assessment ( $p$ $=0.037)$ and worse immediate language outcome $(p=0.029)$. This is mainly due to the changes in the expressive component of language (SST expressive preoperatively $-p=0.045$ ) and SST expressive postoperatively-0.030). No significant changes were identified at the level of the receptive component of language. At the subcortical level, the involvement of the deep white matter of the SFG-MFG was related with worse expressive 


\section{A}

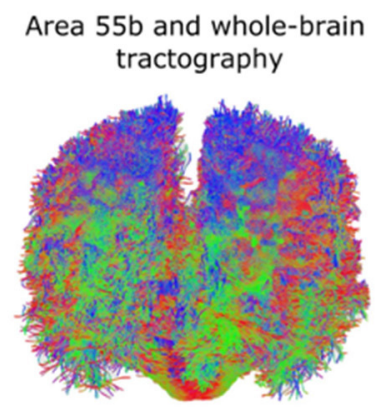

B Area 55b and ROI-based tractography of AF tract

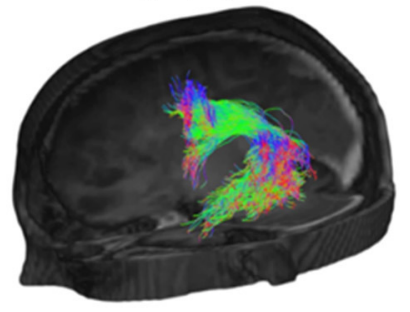

C Whole-brain connectome

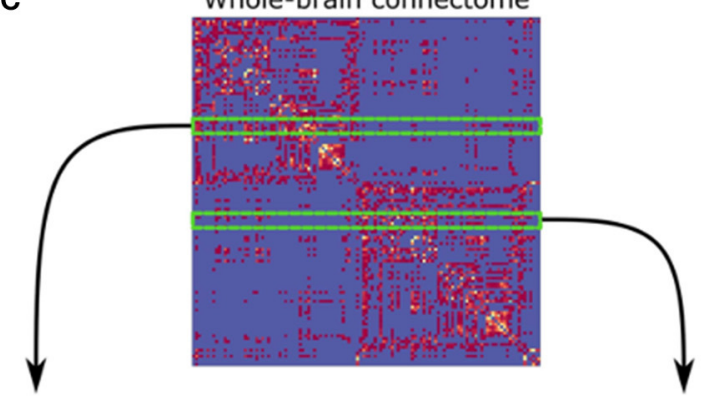

Connectivity from the left Area 55b

to the remaining brain areas

D

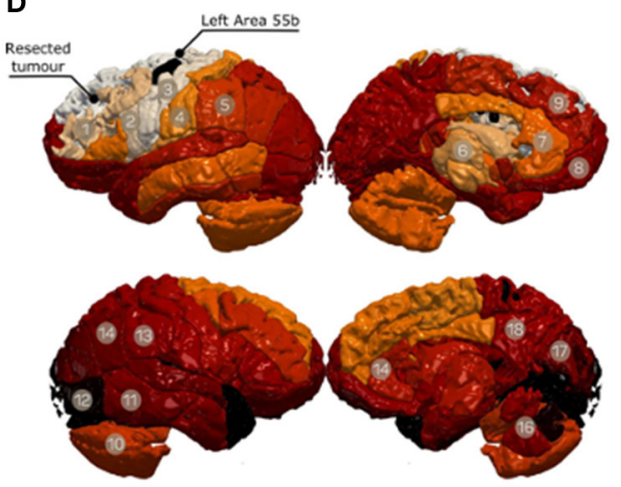

Connectivity from the right Area 55b to the remaining brain areas

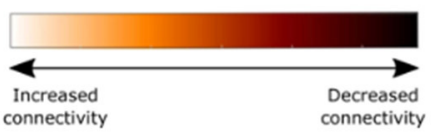

FIGURE 3 | Qualitative analysis for region of interest-based tractography of the arcuate fasciculus (AF). The middle frontal gyrus region is in red, where the streamlines from the AF terminate. (A) Area 55b and whole-brain tractography. (B) Area 55b and ROI-based tractography of AF tract. (C) Whole-brain connectome. (D) Connectivity from the left Area 55b to the remaining brain areas. (E) Connectivity from the right Area 55b to the remaining brain areas.

outcome postoperatively ( $p=0.037$ ), but no correlation was identified with the preoperative assessment $(p=0.780)$, and no overall impact was reflected in the overall SST assessment (SST preoperatively $-p=0.895$; SST postoperatively -0.109 ). The preoperative nTMS mapping was not related with the language outcome (Table 4).

\section{DISCUSSION}

The inferior frontal gyrus and the "classical" Broca's area have been traditionally considered as the main language hub in the dominant frontal lobe. However, the findings from multiple intraoperative reports showed that the functional organization of the frontal lobe is more complex, with positive language sites described both at the level of the MFG and, to a lesser extent, in the SFG (34-39).

In recent years, there has been a renewed interest in the role of the MFG as part of the language network. This is particularly the case after the description of area 55b, located at the posterior aspect of the MFG (7). Previous studies have implicated the role of posterior MFG and area 55b in language $(7,9,10)$. In the present series, we report a high rate of positive speech responses at the level of the MFG ( $45 \%$ of intraoperative errors). 
TABLE 3 | Correlation of navigated transcranial magnetic simulation responses per gyrus and the preoperative language assessment (Sheffield aphasia screening test for acquired language disorder).

\begin{tabular}{lccc}
\hline & Coef. & $\mathbf{9 5 \%} \mathbf{C l}$ & $\boldsymbol{p}$-value \\
\hline nTMS IFG & & & \\
SST & $0.21 \pm 0.7$ & $(0.05-0.38)$ & 0.017 \\
Receptive & $0.35 \pm 0.08$ & $(0.18-0.53)$ & 0.001 \\
Expressive & $0.22 \pm 0.25$ & $(-0.35-0.78)$ & 0.407 \\
nTMS MFG & & & \\
SST & $-0.19 \pm 0.09$ & $(-0.38-0.01)$ & 0.059 \\
Receptive & $-0.29 \pm 0.11$ & $(-0.75-0.45)$ & 0.031 \\
Expressive & $-0.15 \pm 0.26$ & & 0.579 \\
nTMS SFG & & $(-0.19-0.16)$ & 0.877 \\
SST & $-0.01 \pm 0.8$ & $(-0.32-0.18)$ & 0.538 \\
Receptive & $-0.07 \pm 0.11$ & $(-0.33-0.59)$ & 0.538 \\
Expressive & $0.13 \pm 0.20$ & & \\
\hline
\end{tabular}

TABLE 4 | Cortical and subcortical intraoperative involvement and pre- and postoperative language outcomes.

\begin{tabular}{|c|c|c|c|}
\hline & Coef. & $95 \% \mathrm{Cl}$ & $p$-value \\
\hline \multicolumn{4}{|l|}{ Number of gyri involved } \\
\hline SST preoperative & $1.35 \pm 0.65$ & $(0.08-2.62)$ & 0.037 \\
\hline SST postoperative & $-0.66 \pm 0.30$ & $(-1.26$ to -0.69$)$ & 0.029 \\
\hline SST receptive preoperative & $0.25 \pm 0.21$ & $(-0.20-0.69)$ & 0.250 \\
\hline SST receptive postoperative & $-0.11 \pm 0.11$ & $(-0.34-0.12)$ & 0.316 \\
\hline SST expressive preoperative & $0.59 \pm 0.26$ & $(0.01-1.16)$ & 0.045 \\
\hline SST expressive postoperative & $-0.17 \pm 0.07$ & $(-0.32$ to -0.02$)$ & 0.030 \\
\hline \multicolumn{4}{|c|}{ Subcortical involvement - SFG-MFG } \\
\hline SST preoperative & $-0.06 \pm 0.48$ & $(-1.00-0.88)$ & 0.895 \\
\hline SST postoperative & $-0.31 \pm 0.19$ & $(-0.69-0.07)$ & 0.109 \\
\hline SST receptive preoperative & $-0.18 \pm 0.61$ & $(-1.36-1.01)$ & 0.770 \\
\hline SST receptive postoperative & $-0.41 \pm 0.37$ & $(-1.14-0.32)$ & 0.276 \\
\hline SST expressive preoperative & $0.32 \pm 1.14$ & $(-1.91-2.55)$ & 0.780 \\
\hline SST expressive postoperative & $-0.72 \pm 0.34$ & $(-1.40$ to -0.04$)$ & 0.037 \\
\hline \multicolumn{4}{|c|}{ Subcortical involvement - IFG-MFG } \\
\hline SST preoperative & $0.29 \pm 0.48$ & $(-0.64-1.22)$ & 0.544 \\
\hline SST postoperative & $0.24 \pm 0.18$ & $(-0.12-0.60)$ & 0.193 \\
\hline SST receptive preoperative & $0.57 \pm 0.62$ & $(-0.64-1.78)$ & 0.357 \\
\hline SST receptive postoperative & $0.20 \pm 0.33$ & $(-0.46-0.85)$ & 0.557 \\
\hline SST expressive preoperative & $-0.01 \pm 1.13$ & $(-2.23-2.21)$ & 0.992 \\
\hline SST expressive postoperative & $0.66 \pm 0.34$ & $(-0.01-1.33)$ & 0.055 \\
\hline
\end{tabular}

Two points require further discussion: First, the high incidence of positive responses in the MFG were replicated with the use of preoperative nTMS in addition to intraoperative DES. In addition, the majority of positive language sites in the MFG were confined to the posterior aspect of the gyrus, covering the anatomical location of area 55b. We hypothesize that these results can be explained due to the involvement of area $55 \mathrm{~b}$ when stimulating the posterior aspect of the MFG.
This is consistent with previous descriptions of the intraoperative responses obtained at area $55 \mathrm{~b}(9)$.

Second, the responses recorded in the MFG with the combined nTMS and DES were both phonological (hesitations and phonemic errors) and semantic (semantic paraphasias and anomias). The involvement of this area in both semantic processing (12) and speech articulation (40) has been well recognized. The results therefore show that the posterior MFG is likely implicated in both the "dorsal phonological" and the "ventral semantic" streams of language (36). The involvement of the posterior MFG area in both streams of language was also supported at the subcortical level, where again both phonological (speech arrest, hesitation, and fluency disturbance) and semantic disturbances (semantic paraphasia and anomia) were elicited while stimulating the white matter deep to the MFG.

The subcortical areas were divided into two main areas (IFG-MFG and MFG-SFG) as the included tumors all involved more than one subcortical area. The majority of the recorded errors at the subcortical level occurred in the IFG-MFG area (72.4\%). Both the arcuate fasciculus (AF) and the inferior fronto-occipital fasciculus (IFOF) are known to cross deep to the IFG-MFG area, with terminations at the level of the posterior MFG. AF, the main dorsal stream fasciculus, has terminations in the MFG documented by anatomical cadaveric, diffusion imaging, and resting-state fMRI studies. It is reported that up to $56 \%$ of patients can have terminations of the AF in the MFG, particularly the long segment (41-44). From a ventral stream perspective, multiple components of the IFOF were proposed based on anatomical studies (45), DES (46), and diffusion imaging (47). These methods concur that this tract has a termination in the posterior aspect of the MFG and therefore may serve as a substrate for the semantic errors identified in this area (45). The errors detected at the level of the MFG-SFG could be related to the stimulation of the fronto-aslant tract (hesitation and decreased fluency). This tract has been recently involved in the dorsal stream functions of language, particularly the fluency and initiation of speech (48-50).

In addition, the original structural connectivity data presented also support a strong connectivity of the MFG with the adjacent cortical gyri (IFG and SFG), likely mediated via Ufiber short association fibers. These findings are similar to those of other connectivity studies reported in the literature $(7,8)$. In this context, the interaction with the FEF in the anterior/middle frontal gyrus raises the possibility of a potential integration of visual recognition processes with speech production (51).

Therefore, two hypotheses can be formulated to support the interaction of posterior MFG and area 55b with both streams of language: a direct connectivity via relay of some subcomponents of both AF and IFOF and an indirect connectivity via the stimulation of U-fibers to the adjacent gyri (IFG and SMA). In addition to previous imaging and dissection data, recent nTMS data support a strong connectivity of language positive sites via the U-fiber system, supporting the indirect connectivity theory (52). The strong connectivity 
to the primary motor cortex further supports the potential role for hand movement integration in language (43) and the involvement of this area in the articulation and praxis of speech (9).

The impact of positive responses in the area 55b on clinical outcomes is difficult to establish, as these are usually associated with positive responses in either the IFG or SFG. Generally, the overall SST score and each of its components deteriorated temporarily after resection. Thus, the direct involvement of the posterior middle frontal gyrus and area 55b was not related with language outcome. However, the involvement of an increased number of gyri was related with better preoperative SST but with worse postoperative SST scores, particularly due to the receptive component. We believe that neuroplasticity within the language network can be partially responsible for these findings. A higher number of involved gyri may imply the involvement of preoperative adaptive mechanisms to maintain a high level of language function. However, at the same time, they may represent an overall stretched network that has a limited ability to recover from the hit provided by surgical resection and therefore linked to worse language outcomes. This natural process of adaptation has been seen in other systems of the human brain, such as the motor system (53), and further studies are required to ascertain if a similar process may be involved in the language connectivity and network.

There is evidence for language network plasticity in patients, given the intrinsic changes in the intra- and interhemispheric inhibition mechanisms altered by pathological conditions (54). Furthermore, multiple preoperative and intraoperative studies have documented the presence of language network plasticity, particularly in tumors with a long course and natural history, such as low-grade gliomas (55-59).

Despite it being acknowledged that there are different degrees of plasticity potential for different functions of language (60), we hypothesize that an increased number of frontal lobe gyri involved in language may act as a surrogate for the degree of plasticity and adaptation of the language network already present before surgery.

To this regard, preoperative speech mapping with nTMS can play an important role in detecting the extent of involvement of the different frontal gyri in language function, thus providing a useful tool for preoperative counseling. It is crucial to take a patient-centred approach in neuro-oncology in order to meet patient expectations with surgical and oncological treatment (61).

This study has the general limitations of a retrospective cohort study. The most significant one is the incomplete preoperative data for some of the patients included, where the posterior middle frontal gyrus and the area 55b were mapped intraoperatively. However, it provides evidence for the added value of the integration of preoperative advanced mapping and intraoperative language mapping of area $55 \mathrm{~b}$ and further establishes this area within the MFG as a potential relay for both ventral and dorsal streams of language.

\section{CONCLUSION}

This case series suggests that the posterior MFG, including area $55 \mathrm{~b}$, is an important integration cortical hub for both dorsal and ventral streams of language. It demonstrates this area as a cluster of positive responses in the MFG for both preoperative nTMS and intraoperative DES language mapping with a potential impact on language outcomes in dominant frontal lobe surgery.

\section{DATA AVAILABILITY STATEMENT}

The original contributions presented in the study are included in the article/Supplementary Material, further inquiries can be directed to the corresponding author/s.

\section{ETHICS STATEMENT}

Written informed consent was obtained from the individual(s) for the publication of any potentially identifiable images or data included in this article.

\section{AUTHOR CONTRIBUTIONS}

SH and MA: data collection, data analysis, literature review, and manuscript writing. JL: data collection, data analysis, literature review, manuscript writing, and statistical analysis. SP: data collection (nTMS, demographics), and written part of methodology. HW: reviewed speech and language data, written part of methodology. OL, CS, HI, AM, and SO: area 55b seed, connectivity data. JL, AK-V, RG, KA, and $\mathrm{RB}$ : reviewed manuscript. FV: principal manuscript reviewer, innovative concepts and ideas. All authors contributed to the article and approved the submitted version.

\section{FUNDING}

OL was funded by the EPSRC Research Council (EPSRC DTP EP/R513064/1). HI and CS were supported by the EPSRC-funded UCL Centre for Doctoral Training in Medical Imaging (EP/L016478/1).

\section{SUPPLEMENTARY MATERIAL}

The Supplementary Material for this article can be found online at: https://www.frontiersin.org/articles/10.3389/fneur. 2021.646075/full\#supplementary-material

Supplementary Material 1 | Tractography. Diffusion and T1-weighted data were pre-processed using the framework described by Mancini et al. (62). Multi-fiber orientations were estimated using single-shell two-tissue constrained spherical deconvolution using order $I_{\max }=8$ (63). Probabilistic tractography was done using Second-order integration over Fiber Orientation Distributions (iFOD2) (63) and seeding randomly from the white-matter/grey-matter interface, both to reconstruct whole-brain (FOD amplitude cut-off $=0.05$ and total_streamlines $=10$ million) and region-of-interest $(\mathrm{ROI})$ based tractography (FOD amplitude cut-off $=0.05$ and total_streamlines $=1,000)$. $\mathrm{ROI}$-based tractography relied on reconstructing the 
arcuate fasciculus (AF) tract from the left side of the brain based on similar pipeline introduced in (62). The posterior MFG was used to filter the reconstructed AF and its connectivity analysis. Additionally, qualitative results are shown in Figure $\mathbf{3}$. For surgical planning purposes, region of interest based tractography using subcortical anatomical areas was performed as described by Fekonja et al., in

\section{REFERENCES}

1. Brodmann K. Vergleichende Lokalisationslehre der Grosshirnrinde in ihren Prinzipien dargestellt auf Grund des Zellenbaues (English Translation by Garey, L.J.: Brodmann's Localization in the Cerebral Cortex; Smith Gordon, London, 1994), Barth (1909).

2. von Economo CF, Koskinas GN. Die cytoarchitektonik der hirnrinde des erwachsenen menschen. London: Springer (1925).

3. Vogt C, Vogt O. Allgemeinere Ergebnisse unserer Hirnforschung. J Psychol Neurol. (1919) 25:279-462.

4. Hopf A. Distribution of myeloarchitectonic marks in the frontal cerebral cortex in man. J Hirnforschung. (1956) 2:311-33.

5. Nieuwenhuys R. The myeloarchitectonic studies on the human cerebral cortex of the Vogt-Vogt school, and their significance for the interpretation of functional neuroimaging data. Brain Struct Func. (2013) 218:303-52. doi: 10.1007/s00429-012-0460-z

6. Van Essen DC, Glasser MF, Dierker DL, Harwell J, Coalson T. Parcellations and hemispheric asymmetries of human cerebral cortex analyzed on surfacebased atlases. Cerebral Cortex. (2012) 22:2241-62. doi: 10.1093/cercor/bhr291

7. Glasser MF, Coalson TS, Robinson EC, Hacker CD, Harwell J, Yacoub E, et al. A multi-modal parcellation of human cerebral cortex. Nature. (2016) 536:171-8. doi: 10.1038/nature18933

8. Baker CM, Burks JD, Briggs RG, Sheets JR, Conner AK, Glenn CA, et al. A connectomic Atlas of the human cerebrum-chapter 3: the motor, premotor, and sensory cortices. Operat Neurosurg. (2018) 15 (suppl_1), S75-S121. doi: 10.1093/ons/opy256

9. Chang EF, Kurteff G, Andrews JP, Briggs RG, Conner AK, Battiste JD, et al. Pure apraxia of speech after resection based in the posterior middle frontal gyrus. Neurosurgery. (2020) 87:E383-9. doi: 10.1093/neuros/nyaa002

10. Donahue CJ, Glasser MF, Preuss TM, Rilling JK, Van Essen DC. Quantitative assessment of prefrontal cortex in humans relative to nonhuman primates. Proc Natl Acad Sci. (2018) 115:E5183-92. doi: 10.1073/pnas.1721653115

11. De Witt Hamer PC, Robles SG, Zwinderman AH, Duffau H, Berger MS. Impact of intraoperative stimulation brain mapping on glioma surgery outcome: a meta-analysis. J Clin Oncol. (2012) 30:2559-65. doi: 10.1200/JCO.2011.38.4818

12. Sarubbo S, Tate M, De Benedictis A, Merler S, Moritz-Gasser S, Herbet G, et al. Mapping critical cortical hubs and white matter pathways by direct electrical stimulation: an original functional atlas of the human brain. Neuroimage. (2020) 205:116237. doi: 10.1016/j.neuroimage.2019.116237

13. Mandonnet E, Winkler PA, Duffau H. Direct electrical stimulation as an input gate into brain functional networks: principles, advantages and limitations. Acta Neurochirurgica. (2010) 152:185-93. doi: 10.1007/s00701-009-0469-0

14. Borchers S, Himmelbach M, Logothetis N, Karnath HO. Direct electrical stimulation of human cortex - the gold standard for mapping brain functions? Nat Rev Neurosci. (2011) 13:63-70. doi: 10.1038/nrn3140

15. Pallud J, Zanello M, Kuchcinski G, Roux A, Muto J, Mellerio C, et al. Individual variability of the human cerebral cortex identified using intraoperative mapping. World Neurosurg. (2018) 109:e313-7. doi: 10.1016/j.wneu.2017.09.170

16. Forster M-T, Hattingen E, Senft C, Gasser T, Seifert V, Szelényi A. Navigated transcranial magnetic stimulation and functional magnetic resonance imaging: advanced adjuncts in preoperative planning for central region tumors. Neurosurgery. (2011) 68:1317-25. doi: 10.1227/NEU.0b013e31820b528c

17. Picht T, Schmidt S, Brandt S, Frey D, Hannula H, Neuvonen T, et al. Preoperative functional mapping for rolandic brain tumour surgery: comparison of navigated transcranial magnetic stimulation to direct cortical stimulation. Neurosurgery. (2011) 69:581-8. Discussion 588. doi: 10.1227/NEU.0b013e3182181b89
(64). The nTMS responses were overlayed over the dissected tracts considered for language network.

Supplementary Video 1 | Preoperative language mapping (nTMS assessment) demonstrating language errors.

18. Picht T, Frey D, Thieme S, Kliesch S, Vajkoczy P. Presurgical navigated TMS motor cortex mapping improves outcome in glioblastoma surgery: a controlled observational study. J Neurooncol. (2016) 126:535-43. doi: 10.1007/s11060-015-1993-9

19. Krieg SM, Sabih J, Bulubasova L, Obermueller T, Negwer C, Janssen I, et al. Preoperative motor mapping by navigated transcranial magnetic brain stimulation improves outcome for motor eloquent lesions. Neurooncology. (2014) 16:1274-82. doi: 10.1093/neuonc/nou007

20. Raffa G, Scibilia A, Conti A, Ricciardo G, Rizzo V, Morelli A, et al. The role of navigated transcranial magnetic stimulation for surgery of motor-eloquent brain tumors: a systematic review and metaanalysis. Clin Neurol Neurosurg. (2019) 180:7-17. doi: 10.1016/j.clineuro.2019.03.003

21. Raffa G, Picht T, Scibilia A, Rösler J, Rein J, Conti A, et al. Surgical treatment of meningiomas located in the rolandic area: the role of navigated transcranial magnetic stimulation for preoperative planning, surgical strategy, and prediction of arachnoidal cleavage and motor outcome. J Neurosurg. (2019) 14:1-12. doi: 10.3171/2019.3.JNS183411

22. Sollmann N, Picht T, Mäkelä JP, Meyer B, Ringel F, Krieg SM. Navigated transcranial magnetic stimulation for preoperative language mapping in a patient with a left frontoopercular glioblastoma. J Neurosurg. (2013) 118:1759. doi: 10.3171/2012.9.JNS121053

23. Krieg SM, Lioumis P, Mäkelä JP, Wilenius J, Karhu J, Hannula H, et al. Protocol for motor and language mapping by navigated TMS in patients and healthy volunteers; workshop report. Acta Neurochirurgica. (2017) 159:118795. doi: 10.1007/s00701-017-3187-z

24. Jeltema HR, Ohlerth AK, de Wit A, Wagemakers M, Rofes A, Bastiaanse $\mathrm{R}$, et al. Comparing navigated transcranial magnetic stimulation mapping and "gold standard" direct cortical stimulation mapping in neurosurgery: a systematic review. Neurosurg Rev. (2020). doi: 10.1007/s10143-020-01397-x

25. Bährend I, Muench MR, Schneider H, Moshourab R, Dreyer FR, Vajkoczy P, et al. Incidence and linguistic quality of speech errors: a comparison of preoperative transcranial magnetic stimulation and intraoperative direct cortex stimulation. J Neurosurg. (2020) 29:1-10. doi: 10.3171/2020.3.JNS193085

26. Krieg SM, Tarapore PE, Picht T, Tanigawa N, Houde J, Sollmann N, et al. Optimal timing of pulse onset for language mapping with navigated repetitive transcranial magnetic stimulation. Neuroimage. (2014) 100:219-36. doi: 10.1016/j.neuroimage.2014.06.016

27. Raffa G, Quattropani MC, Germanò A. When imaging meets neurophysiology: the value of navigated transcranial magnetic stimulation for preoperative neurophysiological mapping prior to brain tumor surgery. Neurosurg Focus. (2019) 47:E10. doi: 10.3171/2019.9.FOCUS19640

28. Syder D, Body R, Parker M, Boddy M. Sheffield Screening Test for Acquired Language Disorders. Windsor: NFER-NELSON (1993).

29. Simpson F, Christie J, Mortensen L, Clark W. Mount Wilga High Level Language Test: Administration \& Scoring Manual Plus Test Form with UK Adaptations and Large Print Additions. (2006). Available online at: http:// nebula.wsimg.com/5dc06d53fe8a246679ba15f02e226ed0?AccessKeyId= 5861B1733117182DC99B\&disposition=0\&alloworigin $=1$

30. Penfield W, Boldrey E. Somatic motor and sensory representation in the cerebral cortex of man as studied by electrical stimulation. Brain. (1937) 60:389-443. doi: 10.1093/brain/60.4.389

31. De Witte E, Satoer D, Robert E, Colle H, Verheyen S, Visch-Brink E, et al. The Dutch linguistic intraoperative protocol: a valid linguistic approach to awake brain surgery. Brain Lang. (2015) 140:35-48. doi: 10.1016/j.bandl.2014.10.011

32. Ohlerth AK, Valentin A, Vergani F, Ashkan K, Bastiaanse R. The verb and noun test for peri-operative testing (VAN-POP): standardized language tests for navigated transcranial magnetic stimulation and direct electrical stimulation. Acta Neurochirurgica. (2020) 162:397-406. doi: 10.1007/s00701-019-04159-x 
33. Jung J, Lavrador JP, Patel S, Giamouriadis A, Lam J, Bhangoo R, et al. First United Kingdom experience of navigated transcranial magnetic stimulation in preoperative mapping of brain tumors. World Neurosurg. (2019) 122:e157887. doi: 10.1016/j.wneu.2018.11.114

34. Sanai N, Mirzadeh Z, Berger MS. Functional outcome after language mapping for glioma resection. $N$ Engl J Med. (2008) 358:18-27. doi: 10.1056/NEJMoa067819

35. Sarubbo S, De Benedictis A, Merler S, Mandonnet E, Balbi S, Granieri E, et al. Towards a functional atlas of human white matter. Hum Brain Map. (2015) 36:3117-36. doi: 10.1002/hbm.22832

36. Sarubbo S, De Benedictis A, Merler S, Mandonnet E, Barbareschi M, Dallabona M, et al. Structural and functional integration between dorsal and ventral language streams as revealed by blunt dissection and direct electrical stimulation. Hum Brain Map. (2016) 37:3858-72. doi: 10.1002/hbm.23281

37. Gonen T, Gazit T, Korn A, Kirschner A, Perry D, Hendler T, et al. Intra-operative multi-site stimulation: Expanding methodology for cortical brain mapping of language functions. PLoS ONE. (2017) 12:e0f180740. doi: 10.1371/journal.pone.0180740

38. Duffau H. The error of Broca: from the traditional localizationist concept to a connectomal anatomy of human brain. J Chem Neuroanat. (2017) 89:73-81. doi: 10.1016/j.jchemneu.2017.04.003

39. Rahimpour S, Haglund MM, Friedman AH, Duffau H. History of awake mapping and speech and language localization: from modules to networks. Neurosurg Focus. (2019) 47:E4. doi: 10.3171/2019.7.FOCUS19347

40. Duffau H, Gatignol P, Mandonnet E, Peruzzi P, Tzourio-Mazoyer N, Capelle L. New insights into the anatomo-functional connectivity of the semantic system: a study using cortico-subcortical electrostimulations. Brain J Neurol. (2005) 128 (Pt 4):797-810. doi: 10.1093/brain/awh423

41. Catani M, Jones DK, Ffytche DH. Perisylvian language networks of the human brain. Ann Neurol. (2005) 57:8-16. doi: 10.1002/ana.20319

42. Martino J, De Witt Hamer PC, Berger MS, Lawton MT, Arnold CM, de Lucas EM, et al. Analysis of the subcomponents and cortical terminations of the perisylvian superior longitudinal fasciculus: a fiber dissection and DTI tractography study. Brain Struct Funct. (2013) 218:105-21. doi: 10.1007/s00429-012-0386-5

43. Barbeau EB, Descoteaux M, Petrides M. Dissociating the white matter tracts connecting the temporo-parietal cortical region with frontal cortex using diffusion tractography. Sci Rep. (2020) 10:8186. doi: 10.1038/s41598-020-64124-y

44. Yagmurlu K, Middlebrooks EH, Tanriover N, Rhoton, ALJr. Fiber tracts of the dorsal language stream in the human brain. J Neurosurg. (2016) 124:1396-405. doi: 10.3171/2015.5.JNS15455

45. Sarubbo S, De Benedictis A, Maldonado IL, Basso G, Duffau H. Frontal terminations for the inferior fronto-occipital fascicle: anatomical dissection, DTI study and functional considerations on a multi-component bundle. Brain Struct Funct. (2013) 218:21-37. doi: 10.1007/s00429-011-0372-3

46. Martino J, Brogna C, Robles SG, Vergani F, Duffau H. Anatomic dissection of the inferior fronto-occipital fasciculus revisited in the lights of brain stimulation data. Cortex. (2010) 46:691-9. doi: 10.1016/j.cortex.2009.07.015

47. Wu Y, Sun D, Wang Y, Wang Y. Subcomponents and connectivity of the inferior fronto-occipital fasciculus revealed by diffusion spectrum imaging fiber tracking. Front Neuroanat. (2016) 10:88. doi: 10.3389/fnana.2016.00088

48. Catani M, Mesulam MM, Jakobsen E, Malik F, Martersteck A, Wieneke C, et al. A novel frontal pathway underlies verbal fluency in primary progressive aphasia. Brain J Neurol. (2013). 136 (Pt 8):2619-28. doi: 10.1093/brain/awt163

49. Vergani F, Lacerda L, Martino J, Attems J, Morris C, Mitchell P, et al. White matter connections of the supplementary motor area in humans. J Neurol Neurosurg Psychiatry. (2014) 85:1377-85. doi: 10.1136/jnnp-2013-307492

50. Kinoshita M, de Champfleur NM, Deverdun J, Moritz-Gasser S, Herbet G, Duffau H. Role of fronto-striatal tract and frontal aslant tract in movement and speech: an axonal mapping study. Brain Struct Funct. (2015) 220:3399412. doi: 10.1007/s00429-014-0863-0

51. Genon S, Reid A, Li H, Fan L, Müller VI, Cieslik EC, et al. Preoperative transcranial magnetic stimulation for picture naming is reliable in mapping segments of the arcuate fasciculus. Brain Commun. (2020) 2:fcaa158. doi: 10.1093/braincomms/fcaa158

52. Zhang H, Schramm S, Schröder A, Zimmer C, Meyer B, Krieg SM, et al. Function-based tractography of the language network correlates with aphasia in patients with language-eloquent glioblastoma. Brain Sci. (2020) 10:412. doi: 10.3390/brainsci10070412

53. Zdunczyk A, Schwarzer V, Mikhailov M, Bagley B, Rosenstock T, Picht T, et al. The corticospinal reserve capacity: reorganization of motor area and excitability as a novel pathophysiological concept in cervical myelopathy. Neurosurgery. (2018) 83:810-8. doi: 10.1093/neuros/nyx437

54. Tzourio-Mazoyer N, Perrone-Bertolotti M, Jobard G, Mazoyer B, Baciu M. Multi-factorial modulation of hemispheric specialization and plasticity for language in healthy and pathological conditions: a review. Cortex. (2017) 86:314-39. doi: 10.1016/j.cortex.2016.05.013

55. Raffa G, Quattropani MC, Scibilia A, Conti A, Angileri FF, Esposito F, et al. Surgery of language-eloquent tumors in patients not eligible for awake surgery: the impact of a protocol based on navigated transcranial magnetic stimulation on presurgical planning and language outcome, with evidence of tumor-induced intra-hemispheric plasticity. Clin. Neurol. Neurosurg. (2018) 168:127-39. doi: 10.1016/j.clineuro.2018.03.009

56. Zheng G, Chen X, Xu B, Zhang J, Lv X, Li J, et al. Plasticity of language pathways in patients with low-grade glioma: a diffusion tensor imaging study. Neural Regen Res. (2013) 8:647-54. doi: 10.3969/j.issn.1673-5374.2013.07.009

57. Cirillo S, Caulo M, Pieri V, Falini A, Castellano A. Role of functional imaging techniques to assess motor and language cortical plasticity in glioma patients: a systematic review. Neural Plast. (2019) 2019:4056436. doi: 10.1155/2019/4056436

58. Southwell DG, Hervey-Jumper SL, Perry DW, Berger MS. Intraoperative mapping during repeat awake craniotomy reveals the functional plasticity of adult cortex. J Neurosurg. (2016) 124:1460-9. doi: 10.3171/2015.5.JNS142833

59. Duffau H. Mapping the connectome in awake surgery for gliomas: an update. J Neurosurg Sci. (2017) 61:612-30.

60. van Geemen K, Herbet G, Moritz-Gasser S, Duffau H. Limited plastic potential of the left ventral premotor cortex in speech articulation: evidence from intraoperative awake mapping in glioma patients. Hum Brain Map. (2014) 35:1587-96. doi: 10.1002/hbm.22275

61. Lavrador JP, Ghimire P, Brogna C, Furlanetti L, Patel S, Gullan R, et al. Preand intraoperative mapping for tumors in the primary motor cortex: decisionmaking process in surgical resection. J Neurol Surg A Cent Eur Neurosurg. (2020). doi: 10.1055/s-0040-1709729

62. Mancini M, Casamitjana A, Peter L, Robinson E, Crampsie S, Thomas $\mathrm{DL}$, et al. A multimodal computational pipeline for $3 \mathrm{D}$ histology of the human brain. Sci. Rep. (2020) 10:13839. doi: 10.1038/s41598-020-69 $163-\mathrm{z}$

63. Tournier JD, Smith R, Raffelt D, Tabbara R, Dhollander T, Pietsch M, et al. MRtrix3: A fast, flexible and open software framework for medical image processing and visualisation. Neuroimage. (2019) 202:116137. doi: 10.1016/j.neuroimage.2019.116137

64. Fekonja L, Wang Z, Bährend I, Rosenstock T, Rösler J, Wallmeroth L, et al. Manual for clinical language tractography. Acta Neurochirurgica. (2019) 161:1125-37. doi: 10.1007/s00701-019-03899-0

Conflict of Interest: The authors declare that the research was conducted in the absence of any commercial or financial relationships that could be construed as a potential conflict of interest.

Copyright (c) 2021 Hazem, Awan, Lavrador, Patel, Wren, Lucena, Semedo, Irzan, Melbourne, Ourselin, Shapey, Kailaya-Vasan, Gullan, Ashkan, Bhangoo and Vergani. This is an open-access article distributed under the terms of the Creative Commons Attribution License (CC BY). The use, distribution or reproduction in other forums is permitted, provided the original author(s) and the copyright owner(s) are credited and that the original publication in this journal is cited, in accordance with accepted academic practice. No use, distribution or reproduction is permitted which does not comply with these terms. 